\title{
LEISHMANIOSE CUTANEA EXPERIMENTAL: I - SOBRE A SUSCEPTIBILIDADE DO PRIMATA CEBUS APELLA (CEBIDAE) À INFECÇÃO PELA LEISHMANIA (VIANNIA) LAINSONI SILVEIRA, SHAW, BRAGA e ISHIKAWA, 1987.
}

\author{
Fernando T. Silveira, Ralph Lainson, Jeffrey J. Shaw, Lourdes M. Garcez, Adelson \\ A. Souza, Roseli R. Braga e Edna A. Ishikawa.
}

\begin{abstract}
Foi investigada a susceptibilidade do primata Cebus apella (Cebidae) à infecção experimental pela Leishmania (Viannia) lainsoni, com o objetivo de estudar a patogenia desse parasita, ainda pouco conhecido para o homem. Dessa forma, cinco espécimens jovens daquele primata, 2 machos e 3 fêmeas, foram inoculados, intraderme, em oito sitios diferentes da regiāo dorsal da cauda com $3 \times 10^{6}$ de promastigotas do parasita (MHOM/BR/81/M6426, Benevides, Pará), obtidas de cultura da fase estacionária. Em seguida às inoculaçōes, a infecção experimental nos animais foi comprovada, não só pela presença de amastigotas do parasita na pele dos animais inoculados, mas, também, pela concomitância desse achado associado ao desenvolvimento de lesão cutânea nos pontos da pele onde o parasita foi inoculado. Diante desses resultados, ficou demonstrada a susceptibilidade do primata Cebus apella à infecção experimental pela Leishmania lainsoni, cujo período de infecção durou quase quatro meses, suficiente para testar drogas antileishmanióticas e estudar a patogênese da doença causada por este parasita.
\end{abstract}

Palavras-chaves: Leishmaniose cutânea experimental. Primata Cebus apella. Leishmania (Viannia) lainsoni.

$\mathrm{Na}$ Região Amazônica brasileira, a leishmaniose tegumentar guarda características bastantes peculiares, dentre as quais sobressaem os diferentes aspectos clínicos observados nos pacientes com a doença. Este fato parece estar diretamente relacionado não só ao estado imunológico do homem parasitado, mas, também, ao caráter individual das diferentes espécies de Leishmania causadoras da doença na referida região. Nesse sentido, faz-se importante lembrar que, até recentemente, eram conhecidas três espécies de Leishmania responsáveis pela infecção tegumentar humana: a Leishmania (Viannia) braziliensis (Vianna, 1911), a Leishmania (Viannia) guyanensis Floch, 1954 e a Leishmania (Leishmania) amazonensis Lainson \& Shaw, 1972. Mais recentemente, Silveira e cols ${ }^{10}$ deram conhecimento de uma nova

Instituto Evandro Chagas (Fundação SESP). Av. Almirante Barroso 492 - CP. 1128, 66050 Belém - Pará - Brasil.

Trabalho realizado com apoio da Fundação Serviços de Saúde Pública, MS, Brasil; Wellcome Trust, Londres; e UNDP/WORLD BANK/WHO Special Programme for Research and Training in Tropical Disease.

Este trabalho constitui parte de tese de mestrado de um dos autores (F.T.S.)

Recebido para publicação em 10/2/89. espécie de parasito, a Leishmania lainsoni, isolado de lesões cutâneas de pacientes oriundos do Estado do Pará. Entretanto, por se tratar de uma espécie cujo potencial patogênico para o homem ainda é pouco conhecido, decidimos avaliar a susceptibilidade do primata Cebus apella à infecção experimental pela Leishmania lainsoni, com o intuito de estabelecer um modelo animal satisfatório para o estudo da biologia e da patogenia desse organismo, a fim de que possa servir de base para um melhor conhecimento da infecção causada no homem.

\section{MATERIAL E MÉTODOS}

\section{Animais de experimentação:}

Objetivando avaliar a susceptibilidade do primata Cebus apella (Cebidae) à infecção experimental pela Leishmania lainsoni, foram selecionados cinco Exemplares jovens desta espécie de primata (10-14 meses de idade), sendo 2 machos e 3 fêmeas, com pesos variando de 1.320-1.530 gramas, todos provenientes do Centro Nacional de Primatas (F. SESP), Belém, Pará, onde se reproduzem em cativeiro. Antes de proceder à inoculação do parasita, os animais foram submetidos a testes sorológicos específicos (Imunofluorescência Indireta) e intradérmicos com antígeno de Montenegro, a fim de ser excluida a possibilidade de terem sido previamente infectados por Leishmania. 
Silveira FT, Lainson R, Shaw JJ, Garcez LM, Souza AA, Braga RR, Ishikawa EA. Leishmaniose cutânea experimental: ISobre a susceptibilidade do primata Cebus apella (Cebidae) à infeção pela Leishmania (Viannia) lainsoni Silveira, Shaw, Braga \& Ishikawa, 1987. Revista da Sociedade Brasileira de Medicina Tropical 22: 125-130, Jul-Set, 1989

No Instituto Evandro Chagas (F. SESP), Belém, Pará, onde este experimento foi realizado, os animais eram mantidos em biotério isolado para primatas, sendo guardados em gaiolas individuais, com dimensões de $47 \mathrm{~cm}$ de largura, $72 \mathrm{~cm}$ de altura e $60 \mathrm{~cm}$ de profundidade. A dieta alimentar dos animais consistiu de leite, ovo, frutas e legumes variados, ração balanceada comercialmente chamada "bonzo" e água.

\section{Infecções experimentais:}

O inóculo utilizado para o desenvolvimento das infecções nos animais consistiu de uma suspensão $(0,1 \mathrm{ml})$ contendo $3 \times 10^{6}$ de formas promastigotas de Leishmania lainsoni (MHOM/BR/M6426, Benevides, Pará), obtidas do $8^{\circ}$ dia de cultivo em meio Difco-B45 11 , a partir de tecido de hamster infectado com o parasita. Desse modo, os cinco animais foram inoculados, intradermicamente, na região dorsal da cauda, previamente depilada, em oito sitios diferentes, recebendo portanto cada animal uma carga total de 24 $\times 10^{6}$ promastigotas.

Para o controle do desenvolvimento das infecçōes experimentais dois hamsters foram inoculados, intradermicamente, nas patas posteriores com a mesma quantidade ( $3 \times 10^{6}$ de promastigotas) do inóculo utilizado para os primatas.

\section{Parâmetros de avaliação da evolução das infecções:}

Posteriormente à inoculaçāo do parasita, os animais eram examinados semanalmente, com o objetivo de serem observados o aparecimento e o desenvolvimento de lesão nos sitios inoculados, permitindo, assim, não só identificar o período de incubação das infecções, mas, também, acompanhar a evolução e a resolução das lesões nos animais. Além disto, os seguintes procedimentos foram utilizados para avaliar o curso das infecções:

a) Biópsias: realizadas a cada 30 dias após as inoculações e utilizadas para a pesquisa direta do parasita, em esfregaços de aposição corados pelo Giemsa

b) Testes intradérmicos com antígeno de Montenegro: realizados a cada 30 dias após as inoculaçōes, visando o desenvolvimento da resposta imune mediada por células. $O$ antígeno utilizado para os testes foi preparado com promastigotas de cultura da $L$. lainsoni (MHOM/BR/81/M6426, Benevides, Pará), de acordo com método descrito por Shaw e Lainson ${ }^{8}$. As injeções intradérmicas eram feitas com $0.1 \mathrm{ml}$ do antigeno $\left(10 \times 10^{6}\right.$ promastigotas $\left./ \mathrm{ml}\right)$, na face anterior do antebraço dos animais e a leitura realizada após 4872 horas. Reações formadas de eritema e/ou de induração maiores que $0,5 \mathrm{~cm}$ foram consideradas positivas.

\section{RESULTADOS}

A primeira indicação do aparecimento de lesão nos animais foi observada 15 dias após as inoculações, ocasião em que foram vistas áreas focais de eritema em mais da metade dos pontos de inoculação do parasita, principalmente nos animais de número 3, 4 e 5, nos quais estas lesões pareciam mais evidentes do que nos animais de número 1 e 2. Uma semana depois, já era possivel identificar algumas lesões eritemato-papulosas nos animais 3,4 e 5, enquanto que nos animais 1 e 2 , as lesões presentes ainda conservavam um caráter eritematoso, porém, bem mais acentuado do que o verificado anteriormente. No trigésimo dia, o quadro lesional nos animais era constituído, predominantemente, por lesões eritèmato-papulosas, tipicamente semelhantes à lesão cutânea observada na fase inicial da infecção leishmaniótica no homem (Figura 1). Associado a este fato, foi encontrada considerável quantidade de formas amastigotas nos esfregaços obtidos das biópsias de lesões cutâneas. Ainda nesta ocasião, o teste intradérmico com leishmanina mostrou-se negativo em todos os animais.

No segundo mês de observação, o desenvolvimento das lesões foi, mais uma vez, notadamente mais acentuado nos animais 3,4 e 5 , cujo pico de crescimento das lesões foi observado em torno de 60 dias após as inoculações, quando estes animais apresentavam lesões túbero-nodulares em quase todos os sítios inoculados. Ao contrário disto, o desenvolvimento das lesões nos animais 1 e 2 foi bem menos evidente, em especial no animal de número 2 que, no final do mesmo periodo (60 dias), apresentava apenas duas lesões, enquanto que o de número 1 apresentava quatro lesões (Tabela 1). Entretanto, a despeito das diferenças observadas no curso das infecções, a pesquisa de Leishmania nas lesões mostrou-se positiva em todos os animais, mesmo naqueles ( 1 e 2) em que as infecções foram menos intensas. Por outro lado, a resposta ao teste intradérmico de Montenegro manteve-se negativa nos cinco animais, tal como o verificado um mês após as inoculações.

No prosseguimento da avaliação, foi constatada, durante o terceiro mês das infecções experimentais, progressiva regressão das lesões nos animais, o que, sem dúvida, ocorreu de forma mais evidente no Cebus de número 2, o qual, após 90 dias de inoculação, já se apresentava curado das lesões antes observadas. Nos outros animais, o processo de resolução das lesões foi mais lento, havendo cura apenas parcial das lesões ao fim do mesmo período de observação (90 dias), quando ainda existiam lesões residuais de aspecto eritemato-infiltrado em alguns pontos de inoculação do parasita. Nessa oportunidade, a pesquisa do parasita nas lesões foi positiva somente no Cebus 1 , e o 
Silveira FT, Lainson R. Shaw JJ. Garcez LM, Souza AA, Braga RR, Ishikawa EA Leishmaniose cutânea experimental: I Sobre a susceptibilidade do primata Cebus apella (Cebidae) a infeccao pela Leishmania (Viannia) lainsoni Silveira. Shan Braga \& Ishikaw'a. 1987. Revista da Sociedade Brasileira de Medicina Tropical 22: 125-130, Jul Set. 1989
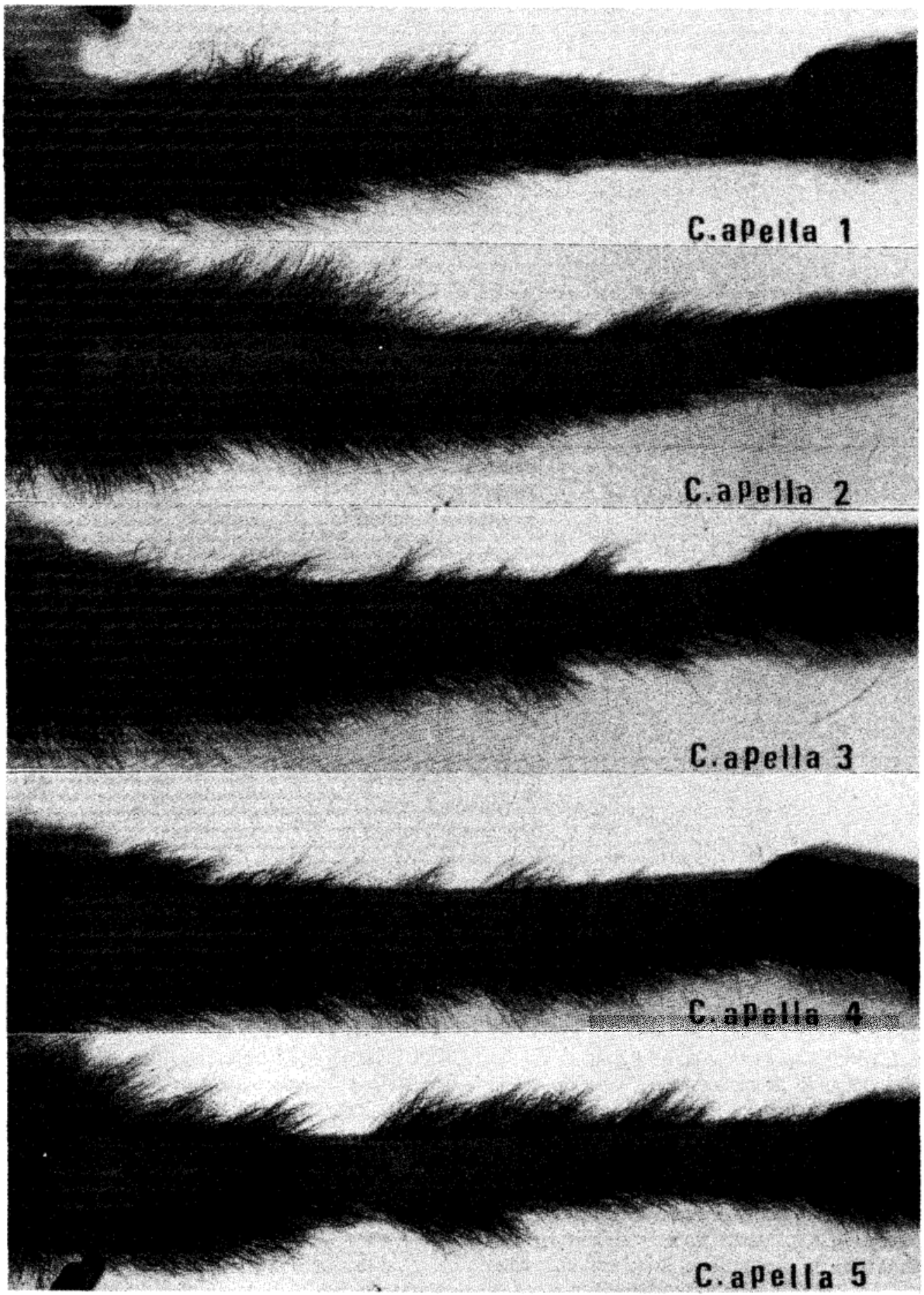

Figura 1 - Notar a presenca de lesōes eritemato-papulosas (indicadas pelas setas), observadas 30 dias após a inoculaçâo da Leishmania (V.) lainsoni na regiào dorsal da cauda do Cebus apella. 
Silveira FT, Lainson R, Shaw JJ, Garcez LM, Souza AA, Braga RR, Ishikawa EA. Leishmaniose cutânea experimental: ISobre a susceptibilidade do primata Cebus apella (Cebidae) à infecção pela Leishmania (Viannia) lainsoni Silveira, Shaw, Braga \& Ishikawa, 1987. Revista da Sociedade Brasileira de Medicina Tropical 22: 125-130. Jul-Set, 1989

Tabela 1 - Aspectos evolutivos da infecção experimental do primata Cebus apella pela Leishmania lainsoni.

\begin{tabular}{|c|c|c|c|c|c|}
\hline $\begin{array}{c}\text { Animal } \\
\text { n. }^{\circ}\end{array}$ & $\begin{array}{l}\text { Periodo P.I.* } \\
\text { (em meses) }\end{array}$ & No de lesões & Tipo de lesão & $\begin{array}{l}\text { Pesquisa do } \\
\text { parasita } \\
\text { (Ex. direto) }\end{array}$ & R.I.M. \\
\hline \multirow{5}{*}{1} & 19 & 6 & Eritêmato-papulosa & + & - \\
\hline & $2 \%$ & 4 & Túbero-nodular & + & - \\
\hline & $3 \circ$ & 3 & Eritèmato-infiltrada & + & - \\
\hline & 40 & 0 & - & & - \\
\hline & $5 \circ$ & 0 & - & & - \\
\hline \multirow{5}{*}{2} & $1 \%$ & 6 & Eritemato-papulosa & + & - \\
\hline & $2 \%$ & 2 & Túbero-nodular & + & - \\
\hline & $3 \%$ & 0 & - & - & + \\
\hline & $4 \%$ & 0 & - & & + \\
\hline & 50 & 0 & - & & - \\
\hline \multirow{5}{*}{3} & $1 \%$ & 7 & Eritêmato-papulosá & + & - \\
\hline & $2 \%$ & 7 & Túbero-nodular & + & - \\
\hline & $3{ }^{\circ}$ & 4 & Eritèmato-infiltrada & - & + \\
\hline & $4 ?$ & 0 & - & & + \\
\hline & $5 \circ$ & 0 & - & & + \\
\hline \multirow{5}{*}{4} & 10 & 7 & Eritêmato-papulosa & + & - \\
\hline & $2 \%$ & 7 & Túbero-nodular & + & - \\
\hline & 39 & 4 & Eritêmato-infiltrada & - & + \\
\hline & $4 \%$ & 0 & - & & + \\
\hline & $5 ?$ & 0 & - & & + \\
\hline \multirow{5}{*}{5} & 19 & 7 & Eritèmato-papulosa & + & - \\
\hline & $2 \%$ & 7 & Túbero-nodular & + & - \\
\hline & $3 \circ$ & 3 & Eritèmato-infiltrada & - & - \\
\hline & $4 \%$ & 0 & - & & - \\
\hline & $5 \%$ & 0 & - & & - \\
\hline
\end{tabular}

\section{* Pós-inoculação \\ R.I.M. Reação Intradérmica de Montenegro \\ + Positivo \\ - Negativo}

teste de Montenegro revelou os primeiros resultados positivos, verificados nos Cebus 2, 3 e 4.

Continuando as observaçôes, foi visto que a resolução completa das lesões nos animais $1,3,4$ e 5 ocorreu por volta de 120 dias após as inoculações, quando, somente nesta ocasião, não foi mais possivel detectar sinais de lesão na pele destes animais, a não ser naqueles locais anteriormente biopsiados, onde ainda eram vistas pequenas lesões residuais de cicatrização do tecido. Além disto, foi observado, mais uma vez, que o teste intradérmico com leishmanina foi positivo apenas nos Cebus 2, 3 e 4, mantendo-se negativo nos outros animais.

Os animais permaneceram em observação até 150 dias após as inoculações, não sendo percebidas lesões de recidiva nos locais de inoculação do parasita, fato este que sugeriu a cura definitiva das infecções.
Entretanto, diferentemente do que foi observado após 120 dias, o Cebus 2 não reagiu ao teste cutâneo com antígeno de Montenegro, assim como os Cebus 1 e 5. Dessa forma, apenas os animais 3 e 4 mantiveram os resultados positivos ao teste cutâneo, tal como o observado após 90 e 120 dias, respectivamente.

\section{DISCUSSÃO}

Em trabalho recente, Lainson e cols ${ }^{4}$ deram conhecimento à primeira referência de infecção natural do primata Cebus apella por Leishmania, cujo parasita foi isolado da pele, aparentemente integra, de 3 espécimens desse primata, capturados em zona endèmica (Serra dos Carajás, Estado do Pará) de leishmaniose tegumentar na Região Amazônica. Esse achado, sem dúvida, reveste-se da maior importância, 
Silveira FT, Lainson R, Shaw JJ, Garcez LM, Souza AA, Braga RR, Ishikawa EA. Leishmaniose cutânea experimental: ISobre a susceptibilidade do primata Cebus apella (Cebidae) à infecção pela Leishmania (Viannia) lainsoni Silveira, Shaw, Braga \& Ishikawa, 1987. Revista da Sociedade Brasileira de Medicina Tropical 22: 125-130, Jul-Set, 1989

não só por suscitar uma possivel participação dessa especie de primata na epidemiologia da leishmaniose tegumentar na referida região, como também por constituir forte evidência do potencial desse animal em estudos experimentais da leishmaniose humana. Nesse sentido, os resultados observados no presente estudo confirmam, de fato, a indicação acima sugerida, uma vez que foi demonstrada a susceptibilidade do $C$. apella à infecção experimental pela Leishmania lainsoni, traduzida pela presença do parasita na pele dos animais experimentalmente infectados e, também, pela concomitância desse achado com o desenvolvimento de lesã́o cutânea, nos pontos da pele onde o parasita foi inoculado.

Por outro lado, trabalhos anteriores 123579 já haviam evidenciado a susceptibilidade de algumas espécies de primatas neotropicais, entre os quais o $C$. apella, à infecção experimental pelos agentes da leishmaniose tegumentar americana. Entretanto, há que ser enfatizado o fato de que, na maioria dos trabalhos acima citados, pouca atenção foi dada à natureza e à quantidade de parasitas presentes nos inóculos, o que, sem dúvida, assume influência no desenvolvimento das infecções experimentais, particularmente no que se refere ao periodo de incubação das infecções e, também, ao tempo de permanência das lesões nos animais. A título de exemplo, Medina ${ }^{7}$, Silva ${ }^{9}$ e Cuba-Cuba e cols ${ }^{2}$, que trabalharam com inóculos não quantificados de formas amastigotas de leishmânias dermotrópicas, observaram periodos de incubação que variaram de 17 dias a 11 meses, fato este que demonstra a impropriedade do uso de inóculos não quantificados. De outra forma, Lujan e $\mathrm{cols}^{5}$ que utilizaram inóculos excessivamente grandes $\left(2 \times 10^{7}\right)$ de promastigotas da Leishmania panamensis para infectar o Aotus trivirgatus, verificaram período de incubação de apenas 7 dias, período este absolutamente incompativel com o que é observado na infecção humana. Quanto ao tempo de permanência das lesões, Lainson e Shaw ${ }^{3}$, que também usaram inóculos não quantificados de amastigotas, observaram que as lesões cutâneas produzidas por espécies de Leishmania dos grupos braziliensis e mexicana no $C$. apella e no Saimiri sciureus permaneceram por tempos excessivamente prolongados, algumas vezes ultrapassando dois anos de duração, o que, sem dúvida, não acrescenta em muito o objetivo das experiências. Diante desses fatos, achamos oportuno registrar os resultados que obtivemos com o uso de inóculos constituídos de número relativamente pequeno $\left(3 \times 10^{6}\right)$ de formas promastigotas do parasita, porém, o suficiente para produzir respostas altamente significativas nos animais, tais como: a) o periodo de incubação das infecções foi de 15-20 dias, quando surgiram as primeiras lesões nos animais (áreas focais de eritema ou lesões eritêmato-papulosas), fato este que está plenamente de acordo con o periodo de incubação observado na leishmaniose cutânea humana; b) no trigésimo dia após as inoculações, predominavam lesões eritemato-papulosas em todos os animais, lesões estas que, conforme referido anteriormente, em muito se assemelham à lesão cutânea observada na fase inicial da infecção leishmaniótica no homem; c) o tempo de permanência das lesões foi de quase quatro meses (120 dias), em pelo menos 4 dos 5 animais inoculados, periodo este suficiente para testar drogas antileishmanióticas e, ainda, coletar material dos animais (biópsias de lesões cutâneas e soro) para estudos relativos aos aspectos histopatológicos e imunopatológicos da doença, o que será motivo de publicações futuras, não só no que se refere à infecção do $C$. apella pela Leishmania (V.) lainsoni, mas, também, pela Leishmania (V.) braziliensis e Leishmania (L.) amazonensis, estudo este que se encontra em andamento; e d) dos cinco animais inoculados, três deles (3, 4 e 5) desenvolveram um maior número de lesões (Tabela 1), que também levaram tempo maior para curar espontaneamente, enquanto que os outros dois (1 e 2) manifestaram menor número de lesões, que regrediram mais rapidamente. Este fato foi outro elemento que veio confirmar a vantagem de utilizar inóculos padronizados, uma vez que, desta maneira, foi possivel demonstrar reações individuais de cada animal perante o mesmo inóculo, fato este que também é observado na doença humana.

Outro aspecto que merece consideração neste trabalho diz respeito à avaliação das infecções experimentais em primatas não humanos através do antigeno de Montenegro. A este respeito, Christensen e Vasque ${ }^{1}$ observaram que no Aotus trivirgatus inoculado com a $L$. braziliensis e com a $L$. mexicana, as primeiras reações positivas aparecem cerca de 60 dias após as inoculaçōes, permanecendo dessa maneira até 139 dias, quando foram realizados os últimos testes intradérmicos com leishmanina. Entretanto, há que ser mencionado que um dos dois animais inoculados com $L$. mexicana, não desenvolveu reação até a última ocasião (139 dias pós-inoculação) em que foi testado. Resultados semelhantes foram verificados por Lujan e cols ${ }^{6}$, os quais evidenciaram testes positivos em torno de 60 dias após a inoculação da mesma espécie de primata, oAotus trivirgatus, pela Leishmania panamensis. Notaram, ainda, que as reações tornaram-se mais intensas na fase final das infecçōes experimentais, cerca de 150 dias após as inoculações. No trabalho em questão, acreditamos poder destacar 3 pontos que merecem maiores comentários. $\mathrm{O}$ primeiro, referente ao fato de que as primeiras reações positivas, observadas em três (os de números 2,3 e 4 ) dos cinco animais testados, foram evidenciadas cerca de 90 dias após as inoculações dos animais (Tabela 1), momento este em que foi observada nítida regressão 
Silveira FT, Lainson R, Shaw JJ, Garcez LM, Souza AA, Braga RR, Ishikawa EA. Leishmaniose cutânea experimental: ISobre a susceptibilidade do primata Cebus apella (Cebidae) à infecção pela Leishmania (Viannia) lainsoni Silveira, Shaw, Braga \& Ishikawa, 1987. Revista da Sociedade Brasileira de Medicina Tropical 22: 125-130, Jul-Set, 1989

das lesões, sugerindo intensa atividade da resposta imune mediada por células. O segundo, relacionado ao fatọ de que as reações persistiram positivas até 150 dias após as inoculações, com exceção do Cebus 2 , que nesta ocasião não respondeu ao teste de Montenegro, fato este que talvez encontre explicação face ao rápido processo de cura da infecção observado neste animal. Em última instância, merece ser salientado que os animais 1 e 5 responderam ao teste de Montenegro, mesmo após 150 dias das inoculações, fato este semelhantemente observado por Christensen e Vasquez ${ }^{1}$ na infecção experimental do $A$. trivirgatus pela L. mexicana e que, no momento, não temos argumentos para discutir, a não ser reconhecer as limitaçōes do uso do teste de Montenegro, especialmente em estudos desta natureza e, também, na triagem dos animais para futuras investigações.

Por último, cabe enfatizar, ainda, a vantagem de terem sido utilizados neste experimento animais provenientes de reprodução em cativeiro, o que afasta a inconveniência do uso de animais selvagens, que podem estar previamente sensibilizados por Leishmania.

\section{SUMMARY}

The susceptibility of the monkey Cebus apella (Cebidae) to experimental infection by Leishmania (Viannia) lainsoni has been investigated. For this purpose, five young monkeys, 2 males and 3 females, were intradermally, inoculated, in eight different places along the dorsal surface of the tail with $3 x$ $10^{6}$ promastigotes of the parasite (MHOM/BR/81/M6426, Benevides, Pará), from stationary phase culture in Difco B45 medium. After inoculations, infection in the monkeys was indicated by the presence of amastigotes in the skin lesions produced in these animals at the points of inoculation, confirming the susceptibility of the monkey Cebus apella to experimental infection by Leishmania lainsoni, with an infection period of four months. This represents a suitable period for testing antileishmanial drugs or studying the pathogenesis of the disease caused by this parasite.

Key-words: Experimental cutaneous leishmaniasis. Primate Cebus apella. Leishmania (Viannia) lainsoni.

\section{AGRADECIMENTOS:}

Gostariamos de manifestar nosso agradecimento em especial à pessoa do Sr. Manoel Carmelino, pela sua dedicação e presteza no cuidar dos animais. Aos Drs. José Augusto Muniz e Marco Aurélio Malacco, médicos veterinários responsáveis pelo Centro $\mathrm{Na}$ - cional de Primatas, pelo apoio técnico durante este trabalho. Aos Srs. Antonio Francisco Pires Martins e Raimundo da Cunha Mendonça pelo auxilio na coleta de material dos animais e, também, ao Sr. Aluísio Figueiredo pelo material fotográfico.

\section{REFERÊNCIAS BIBLIOGRÁFICAS}

1. Christensen HA, Vasquez AM de. Susceptibility of Aotus trivirgatus to Leishmania braziliensis and Leishmania mexicana. The Amefican Journal of Tropical Medicine and Hygiene 30: 54-56, 1981.

2. Cuba-Cuba A, Vexenat AN, Marsden PD, Magalhāes, AV, Barreto AC, Thiago de Mello M. Callithrix sp. (Callithricidae) e Leishmania braziliensis braziliensis. Tentativa do desenvolvimento de um modelo de leishmaniose mucosa experimental. In: Resumos do XXII Congresso da Sociedade Brasileira de Medicina Tropical, Belo Horizonte p. 80, 1986.

3. Lainson R, Shaw JJ. Leishmaniasis in Brazil: XII. Observations on cross-immunity in monkeys and man infected with Leishmania mexicana mexicana, $L$. $m$. amazonensis, L. braziliensis braziliensis, L. b. guyanensis and $L . b$. panamensis. The Journal of Tropical Medicine and Hygiene London 80: 29-35, 1977.

4. Lainson R, Shaw JJ, Braga RR, Ishikawa EA, Souza AA, Silveira FT. Isolation of Leishmania from monkeys in the Amazon Region of Brazil. Transactions of the Royal Society of Tropical Medicine and Hygiene 82: 231, 1988.

5. Lujan R, Chapman WL, Hanson WL, Dennis VA. Leishmania braziliensis: Development of primary and satellite lesions in the experimentally infected owl monkey, Aotus trivirgatus. Experimental Parasitology 61: 348-358, 1986.

6. Lujan R, Dennis VA, Chapman Jr WL, Hanson WL. Blastogenic responses of peripheral blood leukocytes from owl monkeys experimentally infected with Leishmania braziliensis panamensis. The American Journal of Tropical Medicine and Hygiene 35: 1103-1109, 1986.

7. Medina R. Leishmaniasis experimental en animales silvestres. Dermatologia Venezolana 5: 91-119, 1966.

8. Shaw JJ, Lainson R Leishmaniasis in Brazil: X. Some observations on intradermal reactions to different trypanosomatid antigens of patients suffering from cutaneous and mucocutaneous leishmaniasis. Transactions of the Society of Tropical Medicine and Hygiene 69: 323-335, 1975 .

9. Silva AA. Susceptibilidade de sagüis do gênero Callithrix Erxleben, 1777 (Primates, Callithricidae) a alguns protozoários parasitas do homem. Tese de Mestrado, Universidade Federal de Minas Gerais, Belo Horizonte, 1974.

10. Silveira FT, Shaw JJ, Braga RR, Ishikawa E. Dermal leishmaniasis in the Amazon region of Brazil: Leishmania (Viannia) lainsoni sp. $n$., a new parasite from the State of Pará. Memórias do Instituto Oswaldo Cruz 82: 289-292, 1987

11. Walton BC, Shaw JJ, Lainson R. Observations on the in vitro cultivation of Leishmania braziliensis. The Journal of Parasitology 63: 1118-1119, 1977. 